\title{
Ultrasound for Critical Care Physicians: Take a Deep Breath
}

\author{
David Ling, DO \\ Michel Boivin, MD
}

Division of Pulmonary, Critical care and Sleep Medicine University of New Mexico School of Medicine

Albuquerque, NM

A 40 year old man with a past medical history of intravenous drug abuse presented to the emergency department with difficulty walking and lower extremity weakness. He did admit to recent heroin use. He became somnolent in the ED and was given naloxone. However, he did not improve his level of consciousness sufficiently and was intubated for hypercarbia. The patient was transferred to the MICU and was evaluated for respiratory failure. He later that day passed a spontaneous breathing trial after he awoke and was extubated. However, he was soon thereafter was re-intubated for poor respiratory efforts and a weak cough.

With an unexplained etiology for the respiratory failure, CT of the head, MRI of the brain and lab evaluation were pursued but were negative. At that point, a bedside ultrasound of the right hemi-diaphragm in the zone of apposition was obtained and is shown below:

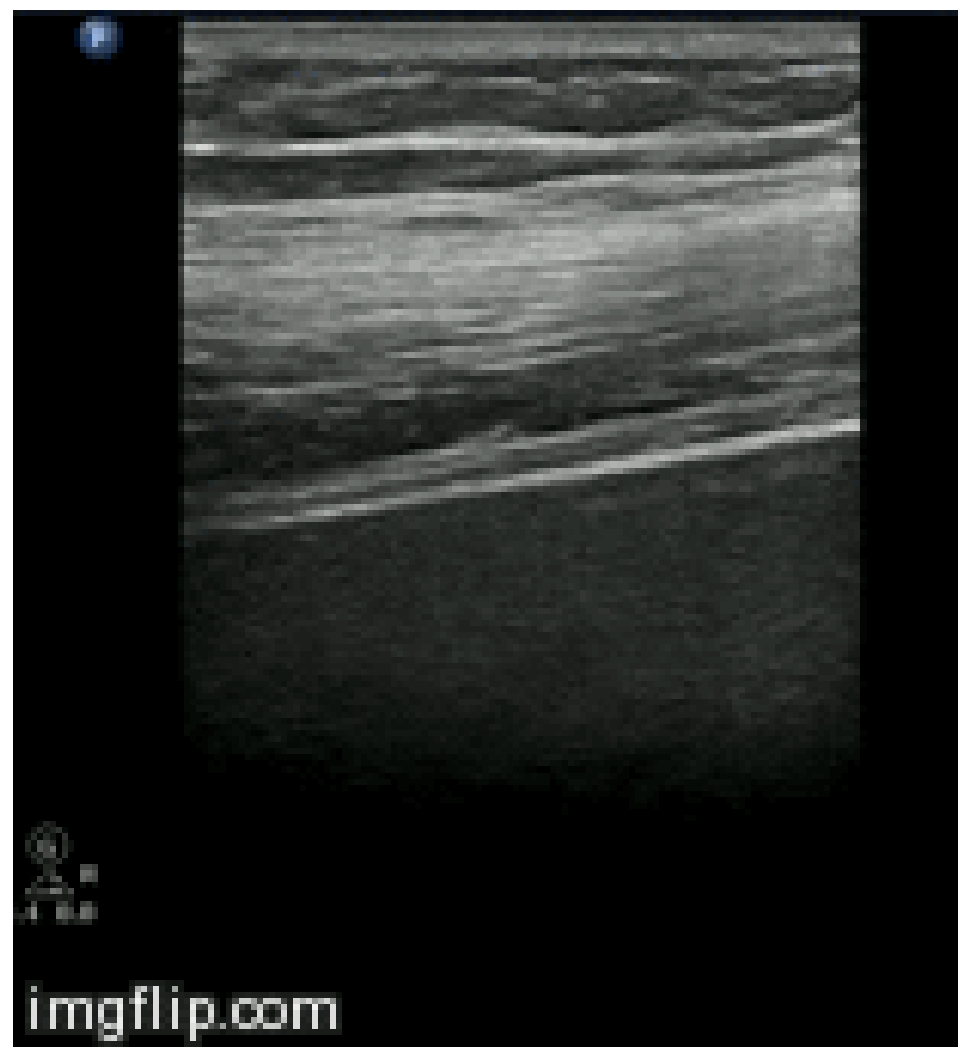

Figure 1. Ultrasound of the right hemi-diaphragm at low depth, at the zone of apposition. The diaphragm is visualized above the liver as three parallel echogenic stripes. 


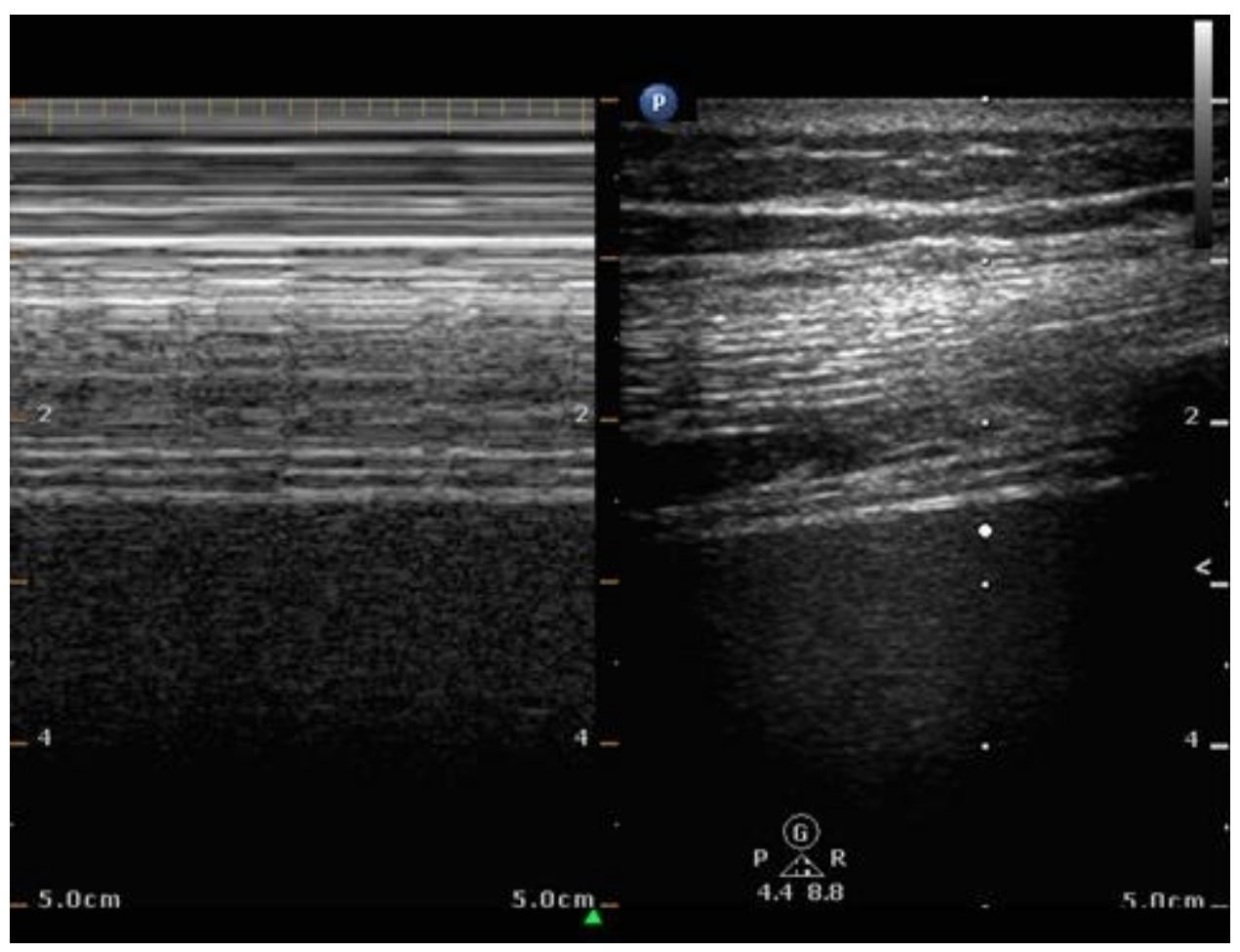

Figure 2. M-mode image of the right hemi-diaphragm. The m-mode image is on the left, and the corresponding $2 \mathrm{D}$ image is on the right.

What does the video and M-mode of the diaphragm demonstrated above predict for the potential result of the patient's extubation?

1. Success

2. Failure

3. Has no predictive value 


\section{Correct!}

\section{Failure}

The video predicts extubation failure. The patient was diagnosed with Guillain-Barré syndrome, based on his neurologic exam and EMG findings. The patient developed prolonged respiratory failure. The clinical course was uneventful post tracheostomy and his care was transferred to a long term acute care facility for further management. The patient has evidence of diaphragmatic weakness on ultrasound. Specifically, the thickening of the diaphragm is measured by the difference in thickness between inspiration (thicker with contraction) and expiration (thinner). Given the absent diaphragmatic movement, extubation failure would be predicted. As a comparison, an ultrasound of normal diaphragmatic function is presented below.

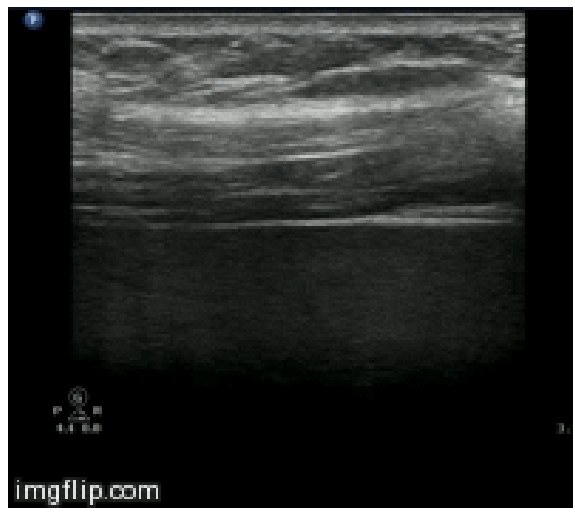

Figure 3. Ultrasound of the right hemi-diaphragm at low depth, at the zone of apposition. The diaphragm thickens in inspiration.

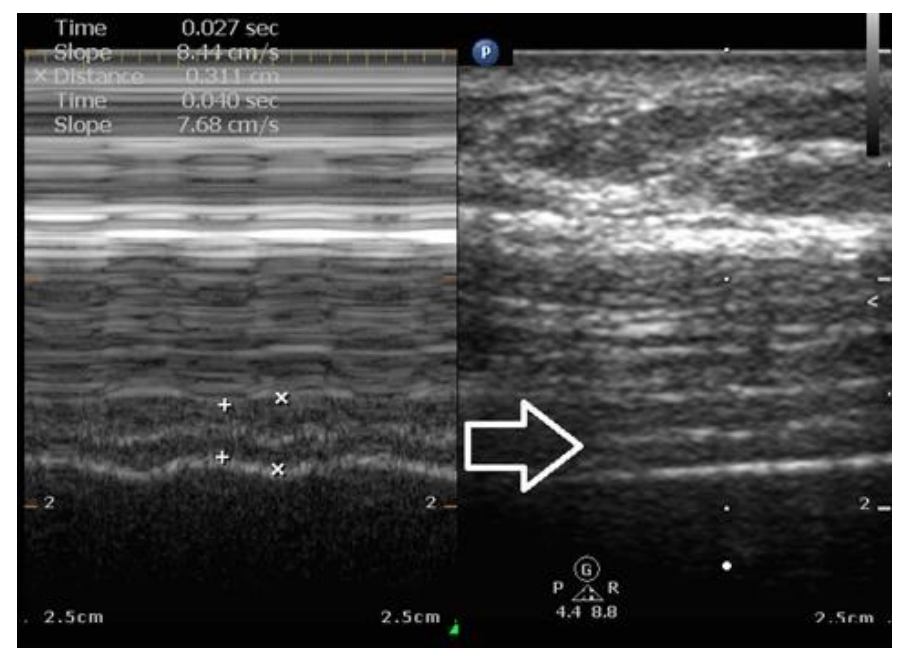

Figure 4. M-mode image of the right hemi-diaphragm. The m-mode image is on the left, and the corresponding to $2 \mathrm{D}$ image is on the right. The diaphragm is visualized above the liver as three parallel echogenic stripes (arrow). The measurements demonstrate a significant increase in thickness with respiration. 
Diaphragm thickening fraction (DTF) assessed by ultrasound is calculated using the following formula:

Thickness at end inspiration - Thickness at end expiration / Thickness at end expiration.

Based on the study published by Ferarri and associates (1), they found that a DTF > $36 \%$ would provide a sensitivity of 0.82 , a specificity of 0.88 , a positive predictive value (PPV) of 0.92 and a negative predictive value (NPV) of 0.75 which was comparable to the standard weaning method of a rapid shallow breathing index of $<105$ (sensitivity of 0.93 , a specificity of 0.88 , a PPV of 0.93 , and a NPV of 0.88 ). This study examined a group of patients with chronic respiratory failure without neuro muscular disease.

The case above demonstrates the usefulness of diaphragmatic thickening in evaluating patients with suspected neuromuscular weakness. While the use of ultrasound to monitor diaphragmatic function is not specifically described in Guillain-Barré syndrome, other methods of determining diaphragm function have been well described in GuillainBarré with respiratory failure (2). It should be noted, bedside ultrasonography is the simplest and least invasive of these tests of diaphragmatic function. The use of ultrasound and the DTF provides an additional tool to assist in the clinical decision making process of ventilator liberation.

\section{References}

1. Ferrari G, De Filippi G, Elia F, Panero F, Volpicelli G, Aprà F. Diaphragm ultrasound as a new index of discontinuation from mechanical ventilation. Crit Ultrasound J. 2014;6(1):8. [CrossRefl [PubMed]

2. Maher J, Rutledge F, Remtulla H, Parkes A, Bernardi L, Bolton CF. Neuromuscular disorders associated with failure to wean from the ventilator. Intensive Care Med. 1995 Sep;21(9):737-43. [CrossRef] [PubMed] 